\title{
EFEITOS DA SUPLEMENTAÇÃO NUTRICIONAL COM AMINOÁCIDOS DE CADEIA RAMIFICADA EM NONAGENÁRIOS E CENTENÁRIOS: PROJETO AMPAL
}

Claudine Lamanna Schirmer. Pontifícia Universidade Católica do Rio Grande do Sul (PUCRS), Escola de Medicina. Porto Alegre, RS, Brasil. E-mail: nutricionistaclaudine@gmail.com Liziane da Rosa Camargo. Pontifícia Universidade Católica do Rio Grande do Sul (PUCRS), Escola de Medicina. Porto Alegre, RS, Brasil. E-mail: lizrcamargo@gmail.com Josemara de Paula Rocha. Pontifícia Universidade Católica do Rio Grande do Sul (PUCRS), Escola de Medicina. Porto Alegre, RS, Brasil. E-mail: josemara.rocha@hotmail.com

Ângelo José Gonçalves Bós. Pontifícia Universidade Católica do Rio Grande do Sul (PUCRS), Escola de Medicina. Porto Alegre, RS, Brasil. E-mail: angelo.bos@pucrs.br

\section{RESUMO}

Introdução: A desnutrição é um problema de saúde que afeta particularmente nonagenários e centenários. Baixa ingesta proteica está associada à piora da funcionalidade, com perda da massa muscular, diminuição da força de membros superiores e inferiores. Objetivo: verificar os efeitos de uma suplementação com aminoácidos de cadeia ramificada em nonagenários e centenários. Métodos: É um ensaio clínico não-randomizado, pareado por conveniência, quantitativo e analítico, com grupo intervenção (GI) que recebeu suplemento alimentar por 3 meses e grupo controle (GC) sem suplemento. Os participantes eram nonagenários e centenários acompanhados pelo Projeto Atenção Multiprofissional ao Longevo (AMPAL). Resultados: O GI teve 10 participantes ( 8 mulheres e 2 homens) e o GC 19 (10 mulheres e 9 homens). A massa magra aumentou no GI em 1,1 $1,69 \mathrm{~kg}$ e peso aumentou $0,6 \pm 2,29 \mathrm{~kg}$, durante o acompanhamento. $\mathrm{O}$ GC apresentou diminuição tanto da massa magra quando no peso total ( $p=0,0804$ ). Ambos os grupos aumentaram a albumina sérica (GI em $0,2 \pm 0,21 \mathrm{mg} / \mathrm{dl}$ e GC em $0,1 \pm 0,26 \mathrm{mg} / \mathrm{dl}$, com $\mathrm{p}=0,4447$ ) e a hemoglobina (GI em $1,3 \pm 0,81 \mathrm{~g} / \mathrm{dl}$ e GC em $0,5 \pm 1,64 \mathrm{~g} /$ $\mathrm{dl}$ com $\mathrm{p}=0,1524)$. Os parâmetros antropométricos aumentaram no GI e diminuíram no GC, sendo significativo para o IMC ( $p=0,0428$ ). A Força de Preensão Palmar e o Timed Up and Go melhoraram no GI e pioraram no GC, embora sem significância estatística ( $p=0,1685$ e $p=0,1797$, respectivamente). Conclusão: Os resultados da presente pesquisa nos permitem concluir que a suplementação por aminoácidos de cadeia ramificada em nonagenários e centenários demonstrou ser eficiente e segura, produzindo melhoras na composição corporal, desempenho funcional e parâmetros bioquímicos.

Palavras-chave: Suplementação nutricional. Longevos. Funcionalidade. Saúde Pública e envelhecimento.

Agradecimentos: O presente trabalho foi realizado com o apoio da Coordenação de Aperfeiçoamento de Pessoal Nível Superior - Brasil (CAPES) - Código de Financiamento 001. 